\title{
$K$ BAND GALAXY COUNTS IN THE SOUTH GALACTIC POLE REGION
}

\author{
T. MINEZAKI ${ }^{1}$, Y. KOBAYASHI ${ }^{2}$, Y. YOSHII ${ }^{3}$, \& B. A. PETERSON ${ }^{4}$ \\ ${ }^{1}$ Kiso Observatory, Institute of Astronomy, The University of \\ Tokyo<minezaki@asterope.mtk.nao.ac.jp> \\ 2 National Astronomical Observatory, Japan \\ $<y a j n$ werope.mtk.nao.ac.jp> \\ 3 Institute of Astronomy, The University of Tokyo \\ $<$ yoshii@omega.mtk.ioa.s.u-tokyo.ac.jp> \\ ${ }^{4}$ Mt. Stromlo and Siding Spring Observatories, The Australian \\ National University <peterson@mso.anu.edu.au>
}

We carried out a $K^{\prime}$ band survey during August and September, 1994, in the south galactic pole region that covers $180.8 \operatorname{arcmin}^{2}$ to $K=19$ and $2.21 \operatorname{arcmin}^{2}$ to $K=21$ by the ANU $2.3 \mathrm{~m}$ telescope at SSO, Australia, equipped with PICNIC, developed at NAOJ. New galaxy number counts from $K=13$ to 22 were obtained, which provided the best determination of the galaxy counts from $K=17.5$ to 19.0 because of our large survey area. They were very consistent with Gardner, Cowie, \& Wainscoat (1993, ApJL, 415, 9) and other observations to $K<19$, however, they were larger than the galaxy counts of Saracco et al. $(1997$, AJ, 114, 887) with similar area and depth of survey around that magnitudes.

We constructed a model of the galaxy counts incorporating the observational biases of the survey, that is, the detection rate and the photometric difference, scatter of galaxies of different redshifts and types estimated by extensive Monte-Carlo simulations. Comparing the model with the "raw" galaxy number counts of the survey, we found that the flat universe with a large cosmological constant, $\left(\Omega_{0}, \lambda_{0}\right)=(0.2,0.8)$ was favored, and that introducing a steep faint end to the galaxy luminosity function produced by late-type galaxies, which was suggested to contribute the excess of faint blue galaxies, had little influence on the number counts. 